\title{
Secuelas neuropsicológicas en pacientes con aneurisma gigante prefrontal poscirugía
}

Neuropsychological sequelas in patient with aneurysm giant prefrontal postcirugia

\author{
César Sarria J. ${ }^{1}$, Victoria llaja R. ${ }^{2}$, Pedro Garcia P. ${ }^{3}$, Luis Álvarez S. ${ }^{4}$ \\ (ReCiBido 20/01/2015, Aceptado 02/04/2016)
}

\begin{abstract}
RESUMEN
El objetivo de la presente investigación es describir y analizar los trastornos de la actividad psíquica personal de pacientes posoperados de aneurisma cerebral prefrontal y explicar el compromiso de su actividad funcional mediante el modelo teórico informacional (Ortiz, 1998). La muestra estuvo conformada por pacientes diagnosticados por un médico especialista, a los cuales les fue extirpado un aneurisma con la particularidad de ser gigante, y que requirió la ablación de un segmento de esta área y otras técnicas neuroquirúrgicas de esta estructura del neocórtex, tan importante para el procesamiento afectivo-emotivo, cognitivoproductivo y conativo-volitivo de la información psíquica consciente del individuo.

El muestreo fue no probabilístico, intencional, y se recogieron los datos durante el periodo de enero del 2009 a mayo del 2010. El diseño fue descriptivo, observacional transversal y retrospectivo, tipo ensayo clínico según la clasificación de Díaz (2009). La población de estudio estuvo constituida por todos los ingresos al servicio de neurocirugía del HNERM (Hospitalización 13 B). Se administró la batería neuropsicológica POC 2005 revisada (Sarria, Llaja \& García, 2012). Para el análisis de los datos se utilizó el SPSS, versión 17, cálculo de frecuencias y porcentajes y otras medidas parámetricas que establecieron el predominio de déficit en los tres componentes del sistema de la personalidad. Se llegó a las siguientes conclusiones:
\end{abstract}

1.- Los pacientes posoperados de aneurisma prefrontal no presentan secuela significativa posterior a la intervención quirúrgica.

2.- En los subtest de ordenamiento de figuras y símbolo-dígitos (WAIS), muestran un desempeño inferior, lo que nos podría indicar que la capacidad de planificación y programación secuencial estaría mermada en su desempeño. No así en los test práxicos (de Luria, ensamblaje de objetos y construcción con cubos), donde el desempeño se encuentra dentro de estándares normales.

3.- Los pacientes posoperados muestran leve trastorno afectivo mixto, tanto depresivo como ansioso, que posiblemente podría explicarse como una reacción de adaptación al cuadro estructural de fondo.

1 Neuropsicólogo HNERM. Docente principal de la Universidad Nacional Mayor de San Marcos.

2 Neuropsicóloga HNERM. Docente de la Universidad Privada César Vallejo y de la UNMSM. E-.mail: Ilaja1@gmail.com.

3 Jefe del Dpto. de Neurocirugía Red Rebagliati. EsSalud. Docente principal de la Facultad de Medicina de la UNMSM.

4 Neuropsicólogo UNMSM Docente asociado de la Universidad Nacional Mayor de San Marcos. 
4.- No tenemos resultados de secuela del ámbito del componente conativo-volitivo, evaluado por algún reactivo específico; sin embargo, a través del método clínico, podemos afirmar que ninguno de nuestros pacientes presentó trastornos a este nivel.

Palabras clave: Actividad psíquica personal, síndrome prefrontal, modelo TIP.

\begin{abstract}
The aim of this investigation is to describe and analyze the conditions of the activity staff psychic patients after prefrontal cerebral aneurysm, and explain the commitment of its functional activity through informational theoretical model (Ortiz,1998). The sample consisted of all patients by the doctor and / diagnostics specialist, which they removed an aneurysm bean that has the distinction of being Giant, who request ablacin a segment this area, and other techniques neurosurg of this important structure of the neocortex for processing affectiveemotional, cognitive and conative volitional production of information psychic aware of the individual. The sampling was not Probabilistic, intentional, and data were collected during the period January 2009 to May 2010. The design was an observational cross-sectional and retrospective essay type signal been rated clinically Daz, M. (2009 UPCH.) The study was constituda poblacin for all admissions to the service of HNERM Neurosurg (Hospitalizacin 13 B) It administered Batery Neuropsycologic revised POC -2005 (Sarria, Llaja \& Garcia, 2012) for the analysis of data it used SPSS, version 17, for the calculation of frequencies and percentages, and other measures pharametrics, to establish the dominance of deficit, in three components of the System of Personality. Arrived to the following conclusions:

1. The prefrontal patient surgery for aneurysm, have no significant sequel after the Intervention quirurgic.

2. In the arrangement of figures subtest symbolo-digits (WAIS) show an unpacking or lower, we may indicate that in this capacity settings planificaciny shall sequential in its he served depleted. Do not grab the test praxic (Luria, object assembly, and construction with blocks.). Where he served standards is within normal.

3. The postoperative patients show mild disorder mixed affective, both depressed and anxious, which may possibly be explained as a reaction to adaptation structural background to the table.

4. We do not have results at the component sequel conative-volitional, rated by some reagent specifically it through of clinic method, we can say that none of our patients present disorders at this level.
\end{abstract}

Keywords: Activity psyquic personal, Sindrome Prefrontal, Model TIP.

\title{
INTRODUCCIÓN
}

El síndrome prefrontal ha sido ampliamente estudiado a nivel internacional en neuropsicología clínica desde la perspectiva luriana (Luria, 1979) según la cual los lóbulos frontales y, en particular, sus formaciones terciarias en el hombre ocupan hasta la cuarta parte de la masa total de los hemisferios cerebrales; no alcanzan la madurez en el niño sino hasta los siete años y son zonas cerebrales responsables del control del estado general del córtex cerebral y del curso de las formas fundamentales y más complejas de la actividad mental humana. Igualmente, la función 
de los lóbulos frontales en la organización del comportamiento ha sido estudiada estrictamente por algunos científicos, como Bechterev (1905), Pavlov (1912) publicado en Anokhin (1949: 94), Pribram entre 1954 y 1960, entre otros citados por Luria et. al., donde se ha demostrado su importancia en la regulación de movimientos y acciones, y en la regulación de las acciones mnésicas e intelectuales.

En la revisión teórica de Ortiz (2008: 55) se nos enuncia que la atención es la forma de organización anticipada que adopta la actividad epiconsciente desde su base cognitiva, y que la motivación y el carácter son procesos psicológicos fundamentales que diferencian al hombre del animal, y, por ende, son los componentes superiores de la conciencia, el componente moral de la personalidad, y una forma de actividad personal regulada por el neocórtex prefrontal. Más aún, nos refiere que la memoria conativo-volitiva es el área de asociación anterior, vale decir, el neocórtex prefrontal dorsolateral. Esta estructura se interconecta con las otras áreas neocorticales afectiva y cognitiva, tanto a través de vías transcorticales e interhemisféricas, como a través de varios núcleos subcorticales del cerebro.

Asimismo, en los síndromes prefrontales son característicos cambios de la personalidad premórbida, indiferencia afectiva y social, depresión, tristeza, ansiedad, irritabilidad, inestabilidad del humor, puerilidad, jocosidad, desinhibición, pérdida de las maneras sociales, estereotipas motoras y del lenguaje, hiperoralidad, megafagia y comportamiento obsesivo-compulsivo. Dentro de los trastornos intelectivos, tenemos falta de iniciativa y toma de decisiones, incapacidad de llevar a cabo una tarea planificada; fallos de atención, concentración y memoria, entre otros; y en casos severos los trastornos pueden llegar a reflejos exagerados de prensión y succión, pérdida del control urinario, pérdida del equilibrio sin coordinación de las extremidades (ataxia frontal) y negligencia motora (Ortiz, 2008).De allí que las afecciones cerebrales a este nivel repercuten notoriamente en la calidad de vida de los individuos y altera su sistema de la personalidad de manera integral.

En lo que se ha encontrado sobre estudios de este síndrome en realidades hispanohablantes, tenemos neurocisticercosis y síndrome prefrontal de Barboza, Sepúlveda \& Montalvo (2007: 28-32), en el que se encontró en cinco casos estudiados en Colombia que la patología principal fue el déficit de atención e hiperactividad.

En nuestra realidad, estos estudios clínicos, donde se intenta realizar el análisis de los trastornos de la actividad psíquica personal post una injuria cerebral, no son frecuentes, más aún si se trata de un estudio posneuroquirúrgico. Este estudio es un análisis neuropsicológico que complementa el estudio realizado por ÁlvarezSimonetti ( 2009: 24). Por analogía, nos hace recordar estudios de otros autores como Luria (1974: 86) sobre heridos de bala en la Segunda Guerra Mundial, por lo que esta investigación constituye un estudio exploratorio-piloto en el campo de la neurociencia aplicada.

Asimismo, es un trabajo inédito con el cual se ha podido realizar una visión aproximada de cómo puede afectarse la calidad de vida de los individuos, y específicamente de nuestros pacientes neuroquirúrgicos; y a partir de allí asumir un objetivo primordial en psicología aplicada a la salud, adoptando medidas de 
prevención y promoción de la salud (Godoy, 1999: 72) dentro del campo de la neuropsicología clínica. Esta es una tarea de vital trascendencia en estos tiempos, ya que las enfermedades neurológicas y neuroquirúrgicas han tenido un incremento en el siglo XXI, con mayor incidencia actual en la población adulta joven y adulta intermedia. Nos planteamos el siguiente problema de investigación: ¿Cuáles son los trastornos de la actividad psíquica personal en pacientes posoperados de aneurisma cerebral prefrontal, y cómo se explica el compromiso de su actividad funcional mediante el Modelo Teórico Informacional de la personalidad (TIP). (Ortiz, 2008: 43).

\section{Objetivos}

1. Estimar cuál de los tres componentes del sistema de la personalidad es el más afectado en pacientes posoperados.

2. Determinar si el desorden del intelecto es el predominante en nuestros pacientes.

3. Corroborar si la evaluación neuropsicológica integral del sistema de la personalidad sigue siendo una medida de diagnóstico válida y confiable para estos desórdenes neuropsicológicos.

\section{Método}

La investigación es de tipo exploratoria. Según la clasificación de Díaz (2009: 22), las hipótesis son de tipo descriptivo. Se empleó un diseño descriptivo, observacional, transversal y retrospectivo. Para la recolección de la muestra, se utilizó un muestreo no probabilístico intencional. Como criterio de inclusión se evaluaron pacientes posoperados de aneurisma gigante prefrontal y fueron excluidos todos aquellos que poseían otra patología neurológica o mental.

\section{Muestra}

Se reclutaron todos los pacientes nuevos que eran hospitalizados en el servicio de neurocirugía con el diagnóstico de aneurisma prefrontal, y que habían sido intervenidos. La evaluación fue posoperatoria, inmediata, en el piso 13 B de neurocirugía.

A continuación, se presentan los datos demográficos de la muestra examinada:

Tabla 1. Sexo

\begin{tabular}{ccc}
\hline & Frecuencia & Porcentaje \\
\hline Varón & 2 & 11,1 \\
\hline Mujer & 16 & 88,9 \\
\hline Total & 18 & 100 \\
\hline
\end{tabular}


Tabla 2. Edad

\begin{tabular}{cccc}
\hline & Máximo & Mínimo & Media \\
\hline $\mathrm{N}=18$ & 78 & 20 & 57.17 \\
\hline
\end{tabular}

\section{Procedimiento}

Se realizaron las coordinaciones previas con los médicos especialistas para que fueran derivados los pacientes a la evaluación neuropsicológica.

Se tomó la anamnesis clínica previa (ver anexo $\mathrm{N}^{\circ} 2$ ) para luego administrar la batería neuropsicológica POC-2005 revisada, en tres sesiones en un ambiente exento de estímulos distractores. Se corroboraron algunos datos con testigos o familiares directos, para tener una aproximación objetiva a su línea basal de la actividad psíquica personal previa a intervención neuroquirúrgica.

Se aplicó el paquete estadístico SPSS versión 17 para el análisis de datos, con estadísticos descriptivos básicos (media y desviación estándar, la t de Student) para la comparación de los hallazgos de los tres componentes estructurales del sistema de personalidad de nuestros pacientes, y otros estadísticos no paramétricos, para analizar si existen correlaciones en el desempeño de los reactivos tipo test de la batería neuropsicológica mencionada.

Asimismo, se realizó la interpretación cualitativa, de corte neuropsicológico bajo el modelo TIP, de los hallazgos neuropsicológicos para contestar las hipótesis propuestas, y arribar a las conclusiones con el rigor metodológico suficiente para que este estudio tenga validez, tanto interna, como recursos de generalización externa.

\section{Hipótesis general}

Los pacientes posoperados de aneurisma prefrontal presentan desórdenes en los tres componentes del sistema de la personalidad.

\section{Hipótesis específicas}

1. Los pacientes posoperados quedan con secuelas irreversibles en los procesos afectivo-emotivos del temperamento.

2. Los pacientes posoperados quedan con secuelas irreversibles prioritariamente en los procesos cognitivo-productivos del intelecto.

3. Los pacientes posoperados quedan con secuelas irreversibles en los procesos conativo-volitivos del carácter.

Se corroboraron algunos datos con testigos o familiares directos, para tener una aproximación objetiva a su línea basal previa a intervención neuroquirúrgica. 


\section{Instrumentos}

Se empleó la batería neuropsicológica POC revisada (en prensa, ver anexo 1), que básicamente es una evaluación integral de la actividad psíquica consciente, donde se puede realizar un análisis cuantitativo y prioritariamente de orden cualitativo personalizado, porque puede ser adaptada a cada paciente de acuerdo a la etiología y a la naturaleza cualitativa del rendimiento en cada prueba. Esta forma de administración de la prueba también se conoce con el nombre de método referido al criterio (MRC), donde se valora el dominio individual de una tarea y, por lo tanto, lo que un individuo puede hacer y no lo que puede hacer una persona comparada con otras personas.

Un ejemplo es el enfoque neurológico de Luria que, en realidad, no es una prueba, sino una estrategia para examinar a los pacientes (la serie de LuriaNebraska fue un intento destinado a lograr que el procedimiento de Luria fuera más estructurado y cuantitativo, pero en el proceso se convirtió en un análisis totalmente diferente).

\section{Resultados}

En la tabla 3, podemos apreciar que trece de los casos evaluados $(\mathrm{N}=18)$ no presentaron deterioro en el subtest de conteo regresivo y dos presentaron deterioro moderado.

Tabla 3. Grado de deterioro en el subtest: conteo regresivo-prefrontal, atención selectiva

\begin{tabular}{ccc}
\hline & Frecuencia & Porcentaje \\
\hline No hay deterioro & 13 & 72,2 \\
\hline Deterioro leve & 3 & 16,7 \\
\hline Deterioro moderado & 2 & 11,1 \\
\hline Total & 18 & 100 \\
\hline
\end{tabular}

La tabla 4 nos muestra que nueve de los sujetos se encuentran en la categoría promedio y tres en la categoría inferior en el test de recuerdo selectivo, que evalúa la memoria verbal.

Tabla 4. Categorías del test de recuerdo selectivo: memoria verbal

\begin{tabular}{ccc}
\hline & Frecuencia & Porcentaje \\
\hline Inferior & 3 & 16,7 \\
\hline Promedio & 9 & 50 \\
\hline Superior & 6 & 33,3 \\
\hline Total & 18 & 100 \\
\hline
\end{tabular}


En la tabla 5, se aprecia que diez de los sujetos presentan un desempeño acorde con su edad cronológica.

Tabla 5. Desempeño en el test de recuerdo de frases

\begin{tabular}{ccc}
\hline & Frecuencia & Porcentaje \\
\hline Desempeño inferior & 5 & 27,8 \\
\hline Desempeño promedio & 10 & 55,6 \\
\hline Desempeño superior & 3 & 16,7 \\
\hline Total & 18 & 100 \\
\hline
\end{tabular}

La tabla siguiente muestra que doce sujetos presentan un desempeño promedio en el recuerdo inmediato de objetos presentados por diez segundos.

Tabla 6. Desempeño en el test de memoria visual de objetos

\begin{tabular}{ccc}
\hline & Frecuencia & Porcentaje \\
\hline Desempeño inferior & 3 & 16,7 \\
\hline Desempeño promedio & 12 & 66,7 \\
\hline Desempeño superior & 3 & 16,7 \\
\hline Total & 18 & 100 \\
\hline
\end{tabular}

En la tabla 7, se puede encontrar una cantidad similar de sujetos entre el desempeño a nivel promedio y el desempeño superior, teniendo un porcentaje de $38,9 \%$ y $33,3 \%$ respectivamente.

Tabla 7. Desempeño en el test de memoria semántica

\begin{tabular}{ccc}
\hline & Frecuencia & Porcentaje \\
\hline Desempeño muy inferior & 1 & 5,6 \\
\hline Desempeño inferior & 4 & 22,2 \\
\hline Desempeño promedio & 7 & 38,9 \\
\hline Desempeño superior & 6 & 33,3 \\
\hline Total & 18 & 100 \\
\hline
\end{tabular}


En la tabla 8 encontramos dos sujetos con desempeño muy inferior a la copia completa de modelos.

Tabla 8. Desempeño en el test de Luria de Apraxia (copia de modelos)

\begin{tabular}{ccc}
\hline & Frecuencia & Porcentaje \\
\hline Desempeño muy inferior & 2 & 11,1 \\
\hline Desempeño inferior & 5 & 27,8 \\
\hline Desempeño promedio & 4 & 22,2 \\
\hline Desempeño superior & 7 & 38,9 \\
\hline Total & 18 & 100 \\
\hline
\end{tabular}

En la siguiente tabla, se observa que tanto el nivel inferior como el normal inferior presentan la misma cantidad de sujetos (frecuencia $=5$ ), lo cual nos indica que el razonamiento lógico se halla por debajo del promedio.

Tabla 9. Niveles de desempeño en subtest de comprensión de situaciones (WAIS)

\begin{tabular}{ccc}
\hline & Frecuencia & Porcentaje \\
\hline Inferior & 5 & 27,8 \\
\hline Normal inferior & 5 & 27,8 \\
\hline Normal promedio & 4 & 22,2 \\
\hline Normal superior & 3 & 16,7 \\
\hline Superior & 1 & 5,6 \\
\hline Total & 18 & 100 \\
\hline
\end{tabular}

En la tabla 10 referida a la construcción con cubos, encontramos que seis de lo sujetos examinados están ubicados en la categoría normal superior, lo que indica que el procesamiento práxico no se encuentra comprometido.

Tabla 10. Desempeño en construcción con cubos (WAIS)

\begin{tabular}{ccc}
\hline & Frecuencia & Porcentaje \\
\hline Inferior & 3 & 16,7 \\
\hline Normal inferior & 4 & 22,2 \\
\hline Normal promedio & 5 & 27,8 \\
\hline Normal superior & 6 & 33,3 \\
\hline Total & 18 & 100 \\
\hline
\end{tabular}

En la tabla siguiente, encontramos que en la mayoría de examinados el desempeño de ordenamiento de figuras es inferior, lo que indica que hay mayor dificultad para resolver este subtest. 
Tabla 11. Desempeño en el subtest de ordenamiento de figuras (WAIS)

\begin{tabular}{ccc}
\hline & Frecuencia & Porcentaje \\
\hline Inferior & 11 & 61,1 \\
\hline Normal inferior & 3 & 16,7 \\
\hline Normal promedio & 3 & 16,7 \\
\hline Normal superior & 1 & 5,6 \\
\hline Total & 18 & 100 \\
\hline
\end{tabular}

En la tabla 12, la mayoría de participantes se ubica en el desempeño normal inferior, lo que indica que en este subtest hay mayor dificultad de resolución.

Tabla 12. Desempeño en ensamblaje de objetos (WAIS)

\begin{tabular}{ccc}
\hline & Frecuencia & Porcentaje \\
\hline Inferior & 4 & 22,2 \\
\hline Normal inferior & 8 & 44,4 \\
\hline Normal promedio & 4 & 22,2 \\
\hline Normal superior & 2 & 11,1 \\
\hline Total & 18 & 100 \\
\hline
\end{tabular}

En la siguiente tabla, la mayoría de casos examinados se ubica en la categoría inferior y normal promedio, presentando ocho de ellos mayor dificultad en la resolución de este subtest.

Tabla 13. Desempeño en el subtest de dígito-símbolos

\begin{tabular}{ccc}
\hline & Frecuencia & Porcentaje \\
\hline Inferior & 8 & 44,4 \\
\hline Normal inferior & 3 & 16,7 \\
\hline Normal promedio & 7 & 38,9 \\
\hline Total & 18 & 100 \\
\hline
\end{tabular}

En la siguiente tabla, podemos observar el desempeño de los examinados en el test de memoria perceptivo-motriz de Benton, notándose que la mayoría no presenta signos de síndrome amnésico, y cuatro de ellos presentan síndrome amnésico leve. 
Tabla 14. Desempeño en el test de Benton

\begin{tabular}{ccc}
\hline & Frecuencia & Porcentaje \\
\hline Síndrome amnésico severo & 1 & 5,6 \\
\hline Síndrome amnésico moderado & 1 & 5,6 \\
\hline Síndrome amnésico leve & 4 & 22,2 \\
\hline Sin síndrome amnésico & 12 & 66,7 \\
\hline Total & 18 & 100 \\
\hline
\end{tabular}

En la siguiente tabla encontramos que en la mayoría de casos, 11 de $\mathrm{N}=18$, no hay disfunción del componente afectivo-emotivo, y cuatro presentan disfunción leve.

Tabla 15. Disfunción del componente afectivo-emotivo (test de Zung)

\begin{tabular}{|c|c|c|c|}
\hline & & Frecuencia & Porcentaje \\
\hline \multirow{5}{*}{ Válidos } & Leve & 4 & 22,2 \\
\hline & Moderada & 1 & 5,6 \\
\hline & Severa & 1 & 5,6 \\
\hline & No hay & 11 & 61,1 \\
\hline & Total & 17 & 94,4 \\
\hline Desaparecidos & Sistema & 1 & 5,6 \\
\hline \multicolumn{2}{|c|}{ Total } & 18 & 100 \\
\hline
\end{tabular}

En esta tabla, se aprecia que siete de los dieciocho casos presentaron depresión leve.

Tabla 16. Niveles de depresión en la escala de Zung

\begin{tabular}{|c|c|c|c|}
\hline & & Frecuencia & Porcentaje \\
\hline \multirow{5}{*}{ Válidos } & Leve & 7 & 38,9 \\
\hline & Moderada & 4 & 22,2 \\
\hline & Severa & 1 & 5,6 \\
\hline & No hay & 5 & 27,8 \\
\hline & Total & 17 & 94,4 \\
\hline Desaparecidos & Sistema & 1 & 5,6 \\
\hline \multicolumn{2}{|c|}{ Total } & 18 & 100 \\
\hline
\end{tabular}

En la tabla siguiente, se observa que la mayoría de casos, siete de $\mathrm{N}=18$, presentaron ansiedad leve, y cuatro ansiedad moderada, lo que indica que se trata de un trastorno primario del componente afectivo-emotivo. 
Tabla 17. Niveles de ansiedad en la escala de Zung

\begin{tabular}{|c|c|c|c|}
\hline & & Frecuencia & Porcentaje \\
\hline \multirow{5}{*}{ Válidos } & Leve & 7 & 38,9 \\
\hline & Moderada & 4 & 22,2 \\
\hline & Severa & 1 & 5,6 \\
\hline & No hay & 5 & 27,8 \\
\hline & Total & 17 & 94,4 \\
\hline Desaparecidos & Sistema & 1 & 5,6 \\
\hline \multicolumn{2}{|c|}{ Total } & 18 & 100 \\
\hline
\end{tabular}

En la tabla de correlaciones, encontramos que el subtest de conteo regresivo presenta correlación alta, significativa e inversa al de memoria de dígitos y recuerdo selectivo. En función de memoria de dígitos se halla una correlación alta y significativa con el subtest de recuerdo selectivo de ítems, memoria visual y subtest de ordenamiento de figuras (WAIS). El subtest de recuerdo de ítems presenta correlación alta con los subtest de semejanzas y conteo regresivo, que evalúan atención selectiva. Asimismo, el número de ítems por ensayo guarda correlación alta e inversa con el subtest de dígito-símbolos.

Tabla 18. Correlación de los componentes de la batería aplicada

\begin{tabular}{|c|c|c|c|c|c|c|c|c|c|c|c|c|c|}
\hline & 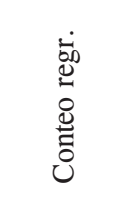 & $\begin{array}{l}.00 \\
=0 \\
0 \\
\Xi 0 \\
\Sigma\end{array}$ & $\begin{array}{l}\overrightarrow{0} \\
\overrightarrow{0} \\
\overrightarrow{\tilde{u}} \\
\ddot{\mathscr{u}}\end{array}$ & 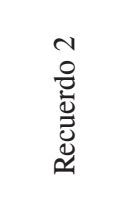 & 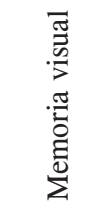 & 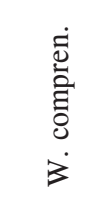 & 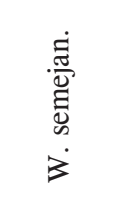 & $\begin{array}{l}\tilde{8} \\
\frac{0}{3} \\
\dot{3}\end{array}$ & 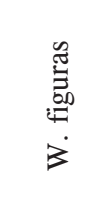 & $\begin{array}{l}0 \\
\frac{0}{0} \\
0 \\
0 \\
3\end{array}$ & 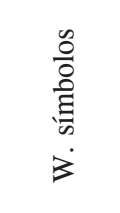 & 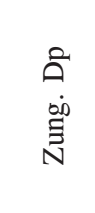 & $\begin{array}{l}\dot{y} \\
\dot{z} \\
\dot{\Xi} \\
\dot{J}\end{array}$ \\
\hline \multicolumn{14}{|c|}{ Correlación de Pearson } \\
\hline Conteo regr. & 1 & $-.725 * *$ & $-.795 * *$ & & $-.598 * *$ & $-.533 *$ & & & & & $-.486^{*}$ & & \\
\hline Memoria dig. & $-.725^{* *}$ & 1 & $.646^{* *}$ & & $.668^{* *}$ & $.544^{*}$ & $.588^{*}$ & & $.604 * *$ & & $.552 *$ & & \\
\hline Recuerdo1 & $-.795 * *$ & $.646^{* *}$ & 1 & & $.587 *$ & $.671 * *$ & $.590 *$ & & & & $.705^{* *}$ & & \\
\hline Recuerdo 2 & & & & 1 & & & $-.670^{* *}$ & & $-.547 *$ & & $-.611 * *$ & $.553 *$ & \\
\hline Memoria vis. & $-.598 * *$ & $.668^{* *}$ & $.587 *$ & & 1 & & & & $.563^{*}$ & & & & \\
\hline W. compr. & $-.533 *$ & $.544 *$ & $.671 * *$ & & & 1 & $.765^{* *}$ & & $.486 *$ & $.621 * *$ & $.856^{* *}$ & & \\
\hline W. semej. & & $.588^{*}$ & $.590 *$ & $-.670 * *$ & & $.765^{* *}$ & 1 & & $.624 * *$ & $.527 *$ & $.922 * *$ & $-.474 *$ & \\
\hline W. cubos & & & & & & & & 1 & & $.573 *$ & & & \\
\hline W. figur. & & $.604 * *$ & & $-.547 *$ & $.563^{*}$ & $.486^{*}$ & $.624 * *$ & & 1 & $.767 * *$ & $.532 *$ & & \\
\hline W. objet. & & & & & & $.621 * *$ & $.527 *$ & $.573 *$ & $.767 * *$ & 1 & $.533 *$ & & \\
\hline
\end{tabular}




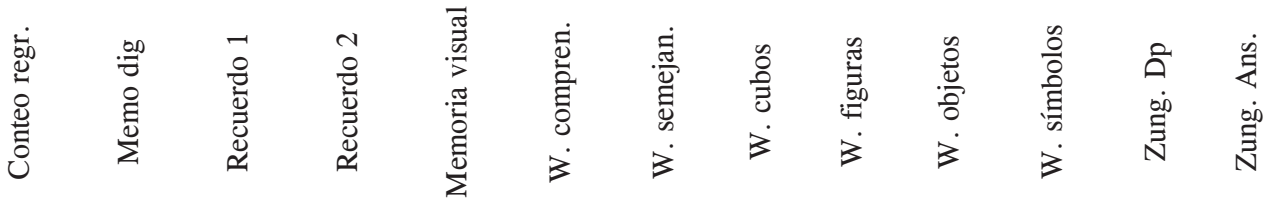

W. símbo. $\quad-.486 * \quad .552 * \quad .705 * * \quad-.611 * * \quad 556 * \quad .922 * * \quad \begin{array}{lllllll} & .532 * & .533 * & 1\end{array}$

\begin{tabular}{|c|c|}
\hline Zung Dp. & $.553 *$ \\
\hline
\end{tabular}

**. Correlation is significant at the 0.01 level (2-tailed).

*. Correlation is significant at the 0.05 level (2-tailed).

\section{DISCUSIÓN}

En el análisis de los datos, encontramos que en la batería neuropsicológica destacan los hallazgos en los subtest de ordenamiento de figuras y símbolo-dígitos del WAIS, que evalúan la capacidad de programación, planificación, y verificación prefrontal. En ellos, se observa un desempeño por debajo del promedio, lo que habla que el déficit encontrado tiene correlación con la ubicación del compromiso estructural, previo a la intervención quirúrgica.

Asimismo, en los test de praxia, tanto en el test de Luria, ensamblaje de objetos, como en la construcción con cubos, no se aprecian dificultades en el desempeño. Más bien, en el primero el desempeño es superior, lo que habla de la conservación de esta actividad.

En los test afectivos se encuentra un trastorno leve, tanto en depresión como en ansiedad, asociado al cuadro de fondo. Este resultado es análogo al encontrado por Martín, Fernández-Armayor, Moreno, García, Rodríguez, Hierro, \& Revilla (1995: 56). A su vez, en el test de retención visual de Benton no se evidencian indicios de compromiso de la memoria a este nivel, lo cual representa un hallazgo no esperado en este síndrome, porque sí fueron encontrados por Sánchez Rodríguez, López Alburqueque \& Sayazo Arenas (1999: 42) en su estudio realizado en España. Probablemente, esto se explica por el éxito del tratamiento neuroquirúrgico, que no afectó la capacidad mnésica visual ni verbal.

En lo que se refiere a las correlaciones, en la mayoría de ellas se observa correlación alta e inversa, lo que demuestra que hay asociación de hallazgos entre los subtest que miden atención selectiva (conteo regresivo), memoria inmediata de dígitos (WAIS), recuerdo selectivo de ítems, memoria visual (nueve elementos visuales), ordenamiento de figuras (WAIS) y semejanzas y dígito-símbolos (WAIS). Esto 
tiene significancia en la medida en que estos subtest están evaluando la actividad propia e intrínseca del lóbulo prefrontal; y con los resultados se puede demostrar que los pacientes de nuestra muestra no han quedado con secuelas significativas posteriores a la intervención quirúrgica realizada, salvo en dos de los subtest ya señalados (ordenamiento de figuras y símbolo dígitos), y a su vez demuestra que nuestros reactivos de la batería son sensibles y útiles para el examen neuropsicológico integral de las lesiones prefrontales de cualquier etiología.

\section{CONCLUSIONES}

1. Los pacientes posoperados de aneurisma prefrontal no presentan secuela significativa posterior a la intervención quirúrgica.

2. En los subtest de ordenamiento de figuras y símbolo-dígitos (WAIS), los pacientes posoperados muestran un desempeño inferior, que nos podría indicar que en capacidad de planificación y programación secuencial estarían mermados. No así en los test práxicos (de Luria, ensamblaje de objetos, y construcción con cubos), donde el desempeño se encuentra dentro de estándares normales.

3. Los pacientes posoperados muestran un leve trastorno afectivo mixto, tanto depresivo como ansioso, que posiblemente podría explicarse como una reacción de adaptación al cuadro estructural de fondo.

4. No tenemos resultados de secuela en el ámbito del componente conativovolitivo, evaluado por algún reactivo específico; sin embargo, a través del método clínico podemos afirmar que ninguno de nuestros pacientes presentó trastornos a este nivel.

\section{RECOMENDACIONES}

Seguir investigando esta casuística neuropsicológica, aplicando reactivos donde se evalúe con mayor exhaustividad el componente conativo-volitivo de la personalidad de nuestros pacientes; lo cual ampliará la explicación integral de los defectos secuelares en el síndrome prefrontal.

\section{REFERENCIAS}

Álvarez-Simonetti, L. \& col. (2009). Aneurismas cerebrales gigantes. Parte I: Aneurismas cerebrales gigantes. Parte II: Patogénesis, aspectos clínicos, imágenes y evaluación diagnóstica. Revista Peruana de Neurociencias. Revistas Especializadas Peruanas REP. Vol. 1. No 1. Pp. 13-21.

Barboza, M. Sepúlveda, S. \& Montalvo, D. (2007). Neurocisticercosis frontal y déficit de atención. Revista Colombia Médica. Corporación Editora Médica del Valle. Vol. 38, $\mathrm{N}^{\mathrm{o}}$ 1. pp. 21-27. 
Díaz, M. (2009). Curso Taller Avanzado. Técnicas y métodos de Investigación Cientifica. Lima: Manual UPCH.

García, P., Llaja, V., Sarria, C. \& col. (2009). Aptitudes cognitivas y estrategias de memorización secuencial de los alumnos de un Centro Educativo privado de Comas. Revista de Investigación en Psicología. Instituto de Investigaciones Psicológicas de la UNMSM. Vol. 12. N 1. Pp. 45-59.

Godoy, J. (1999). Psicología de la Salud: delimitación conceptual. En: Simón, M. (1999). Manual de Psicología de la Salud. Madrid: Biblioteca Nueva.

Luria, A. (1980). Neuropsicología de la Memoria. Madrid: Blume.

Luria, A. R. (1974). Cerebro en Acción. Barcelona: Fontanella

Llaja,V., Sarria, C.\& García, P. (2009). Evaluación neuropsicológica del delirio y la incidencia de los factores de riesgo en pacientes de Cuidados Intensivos del HNERM. Revista de la Academia Peruana de Neurociencias del Perú. Año 1. Vol. 2, pp. 32-36.

Llaja, V., Sarria, C., \& García, P. (2008). Déficits cognitivos en la enfermedad de Parkinson interpretados bajo el modelo informacional de la Personalidad. Revista de la Academia Peruana de Neurociencias del Perú. Vol. 1. Nº1. Pp. 25-28.

Martín, A., Fernández-Armayor, V., Moreno, J. M., García, M. L., Moreno, J. L., Rodríguez, F. A., Hierro, M.,Medialdea, J. \& Revilla, B. (1995). Neurobiología del córtex frontal e hipótesis sobre su papel como procesador natural del caos determinista. Revista Española de Neurología. Vol. 10. pp. 269-289.

Ortiz, P. (2008). Educación y formación de la Personalidad. Lima: Fondo Editorial de la Universidad de Ciencias y Humanidades.

Ortiz. C. P. (2004). Introducción a la Psicobiología del hombre. Cuadernos de Psicobiología Social. Lima: Talleres Gráficos UNMSM.

Ortiz, C. P. ( 2004). El nivel consciente de la actividad personal. Cuadernos de Psicobiología Social. Lima: Talleres Gráficos UNMSM.

Ortiz, C. P. (1998). El nivel consciente de la memoria. Lima: Fondo Editorial de la Universidad de Lima.

Ortiz, P. (1996). La formación de la personalidad. Lima: Dimaso Editores.

Ortiz, P. (1994). El sistema de la personalidad. Lima: UNMSM.

Reynolds, C. R. \& Hickman, J. A. (2004). Draw-A-Person Intellectual Ability Test for 
Sánchez Rodríguez, J., López Alburqueque, J. \& Sayazo Arenas A. (1999). Estudio de un caso prefrontal. Anales de Psicología. Servicio de Publicaciones de la Universidad de Murcia. Vol. 15. No 2. pp. 291-301.

Sarria,C., Llaja,V., \& García, P. (2012). Batería Neuropsicológica POC 2005 Revisada Sets de Evaluación Neuropsicológica del Examen especializado de la Actividad Psíquica personal. Lima: UNMSM.

Sarria, C, García, P., \& Llaja, V. (2008). Compendio de Neuropsicología clínica. Pesquisas Neuropsicológicas. Lima: UNMSM.

Sarria, C., García, P. \& Llaja, V. (2008). Test de Percepción Visomotor de Benton. Revisado. Lima: UNMSM.

\section{ANEXOS}

Anexo $\mathrm{N}^{\mathrm{o}} 1$

Ficha técnica

Nombre del instrumento: Batería neuropsicológica POC 2005 revisada.

Autores: Sarria, C., Llaja, V., \& García, P.

Adaptación: Sarria, C. \& Llaja, V. EsSalud, HNERM. Lima, Perú, año 2005.

Procedencia: Servicio de neurocirugía y unidad de neuropsicología HNERM.

Tipo de prueba: De lápiz y protocolos de examen. Evaluación de orden prioritariamente cualitativo. Emplea el método clínico y tiene, a su vez, validez y confiabilidad desde el punto de vista cuantitativo.

Tiempo: Variable, pero una estimación general es de 45 minutos, en tres sesiones.

Finalidad: Evaluación integral de la actividad psíquica personal, en sus tres componentes: temperamento, intelecto y carácter.

Los test de la batería neuropsicológica están estructurados teniendo como lineamiento base el modelo TIP (Ortiz, 1994 a 2010), con sus distintos planos de actividad: examen de la actividad consciente, examen de la estructura de la conciencia y examen del procesamiento consciente. 